\title{
SPATIAL DISTRIBUTION OF CHROMOSOMES 1 AND Y IN HUMAN SPERMATOZOA
}

\author{
J. P. M. GERAEDTS AND P. L. PEARSON \\ Instituut voor Anthropogenetica, Sylvius Laboratoria der Rijksuniversiteit, \\ Wassenaarseweg 72, Leiden, The Netherlands
}

(Received 3rd April 1975)

\begin{abstract}
Summary. The positions of chromosomes 1 and $\mathrm{Y}$ inside human spermatozoa were determined by differential staining techniques. In $85 / 100$ cells the two chromosomes were in close contact and in association with a vacuole. This observation is in contrast to previous findings for chromosome No. 9 and the Y-chromosome whose positions do not appear to be correlated.
\end{abstract}

Soon after the introduction of the fluorescence staining technique for the detection of the $\mathrm{Y}$ heterochromatin in interphase nuclei (Pearson et al., 1970), it was shown that the Y-chromosome could also be demonstrated in human spermatozoa (Barlow \& Vosa, 1970; Pearson \& Bobrow, 1970) and was associated with vacuoles (Pawlowitzki \& Bosse, 1971). Alternative techniques have now been developed for chromosomes No. 9 (Bobrow et al., 1972) and No. 1 (Geraedts \& Pearson, 1973) which can also be used for demonstrating the heterochromatin of these chromosomes in ejaculated spermatozoa. The staining procedures involved are of the C-banding type, and reveal a darkly stained chromosome body against a less pronouncedly stained nuclear background. In one study (Geraedts \& Pearson, 1975), it was demonstrated that there appeared to be no correlation between the position of the Y-chromosome and chromosome No. 9 . The purpose of the present study was to investigate the spatial relationship between the Y-chromosome and chromosome No. 1 within the human sperm nucleus.

A human ejaculate was washed three times with isotonic saline and the spermatozoa fixed in methanol and kept at $-18^{\circ} \mathrm{C}$. Freshly made, air-dried preparations were stained for chromosome No. 1 (Geraedts \& Pearson, 1973) and examined under oil immersion in green light, without embedding. The slide with the most satisfactory differentiation was selected and slightly destained in methanol (10 min) after rinsing in xylene. The same slide was then restained in $0.5 \%$ atebrine in methanol, rinsed and mounted in distilled water. The quinacrine-stained slides were viewed under a fluorescence microscope, fitted with epi-illumination and a KP 450 exciter filter and a KP 490-nm barrier filter. The positions of chromosomes $\mathrm{Y}$ and 1 were recorded on polaroid photographs of the spermatozoa in green light for the No. 1 chromosome and after 
examination in long-wave u.v. for the $\mathrm{Y}$-chromosome, marking its position on the same photograph. It also proved possible to combine both staining techniques so that by simply switching from u.v. epi-illumination to green light transmission illumination, one could directly go from visualizing one chromosome to another. By this method we could study the relative positions of both chromosomes and observe the generally small but consistent distance between them, i.e. they were associated but not directly superimposed. In some instances the chromosomes were widely separated and this, together with the use of two entirely distinct differential staining methods, leads us to believe that we were not studying the same chromosomes in two different ways.

One hundred cells were photographed, and in 85 chromosome No. 1 was in close contact with the Y-chromosome. In most cells, the chromosomes were lying within the chromatin-dense lower part of the sperm head, close to the boundary of the dense and less-condensed regions (Plate 1). They were often associated with vacuoles, as already observed for the Y-chromosome alone (Pawlowitzki \& Bosse, 1971). Two cells were seen with two No. 1 chromosomes and one with two Y-chromosomes. The frequencies of these disomic cells are in agreement with previous estimates (Pearson et al., 1973). In nondisjunctional cells all three chromosomes were seen in association at the edge of the chromatin-dense region.

This fixed spatial relationship of the heterochromatic regions of chromosomes 1 and $\mathrm{Y}$ in $85 \%$ of spermatozoa is in contrast to our earlier observations for chromosome No. 9 and the Y-chromosome (Geraedts \& Pearson, 1975). Moreover, since chromosomes No. 1 and the $\mathrm{Y}$ are frequently associated with vacuoles, it implies an as yet undefined functional relationship. This is unlikely to be a residual nucleolus association as has been demonstrated for Y-bodies in various types of somatic interphase nuclei (Bobrow et al., 1971).

The arrangement of chromosomes has been studied in the spermatozoa of grasshoppers by $\left[{ }^{3} \mathrm{H}\right]$ thymidine incorporation into sperm precursor cells (Taylor, 1964), and the salamander Plethodon cinereus by the hybridization in situ of an RNA complementary to a particular type of satellite DNA (Walker \& Macgregor, 1975). The position of the Y-chromosome has also been demonstrated in rat spermatozoa by autoradiography (Bianchi \& De Bianchi, 1969) and more recently in the spermatozoa of Microtus oeconomus by a differential staining technique (Tates et al., 1975). Bianchi \& De Bianchi (1969) interpret their results as indicating an end-to-end association of the chromosomes in the sperm nuclei of the rat and also a constant position of the $\mathrm{Y}$-chromosome. In Plethodon cinereus the large (sub)metacentric chromosomes appear to lie parallel to each other, with the centromeres situated at the base of the nucleus. Walker \& Macgregor (1975) conclude that in this species the chromosomes occupy more or less constant positions.

Further research will be needed to see whether other chromosomes also have a non-random position in human spermatozoa. The development of new differential staining techniques or the application of hybridization techniques in situ will be a prerequisite for this study. 
PLATE 1

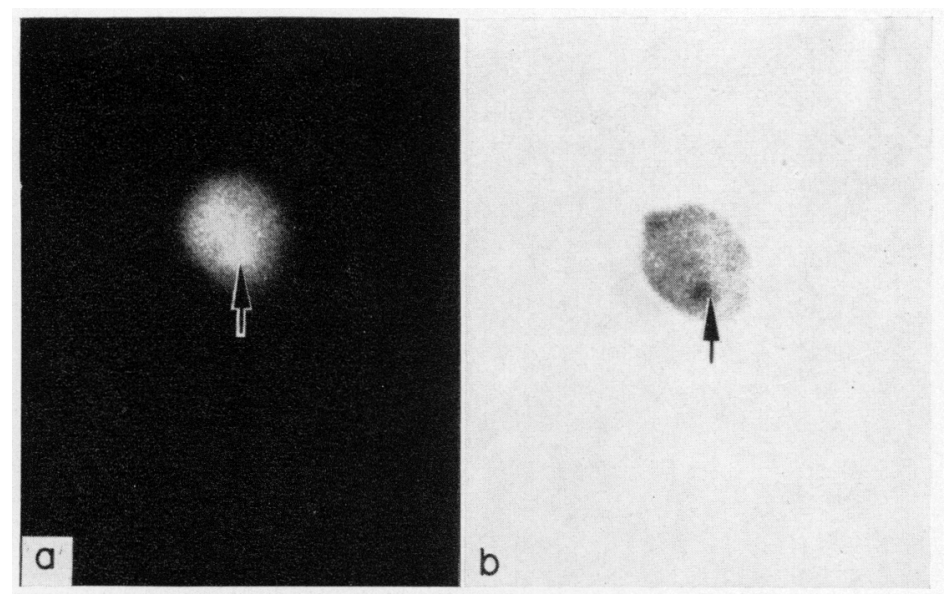

The same human spermatozoon after staining for the Y-chromosome (a) and chromosome No. 1 (b). Note the almost identical position of both chromosomes, indicated by the arrows.

(Facing p. 516) 


\section{REFERENCES}

Barlow, P. \& Vosa, G.G. (1970) The Y chromosome in human spermatozoa. Nature, Lond. 226, 961962.

Branah, N.O. \& De Bianchi, M.S. (1969) Y chromosome replication and chromosome arrangement in germ line cells and sperms of the rat. Chromosoma 28, 370-378.

Bobrow, M., Pzarson, P.L. \& Collacott, H.E.A.G. (1971) Para-nucleolar position of the human Ychromosome in interphase nuclei. Nature, Lond. 232, 556-557.

Bobrow, M., Madan, K. \& Pearson, P.L. (1972) Staining of some specific regions of human chromosomes, particularly the secondary constriction of No. 9. Nature, New Biol. 238, 122-124.

Geraedts, J.P.M. \& Pearson, P.L. (1973) Specific staining of the human No. 1 chromosome in spermatozoa. Humangenetik 20, 171-173.

Geraedts, J.P.M. \& Pearson, P.L. (1975) Chromosomes in human spermatozoa. In Chromosomes Today, Vol. V. Eds P. L. Pearson \& K. R. Lewis. J. Wiley, Jerusalem.

PAwLowItzkr, I.H. \& Bosse, H.G. (1971) Y chromosomes associated with vacuoles in human spermatozoa. Humangenetik 13, 338-340.

Pearson, P.L. \& Bobrow, M. (1970) Fluorescent staining of the Y chromosome in meiotic stages of the human male. F. Reprod. Fert. 22, 177-179.

Pearson, P.L., Bobrow, M. \& Vosa, C.G. (1970) Technique for identifying Y chromosomes in human interphase nuclei. Nature, Lond. 226, 78-80.

Pearson, P.L., Geraedts, J.P.M. \& Pawlowitzki, I.H. (1973) Ghromosomal studies on human male gametes. In Les Accidents Chromosomique de la Reproduction, pp. 219-229. Eds A. Boué \& C. Thibault. INSERM, Paris.

TAYLOR, J.H. (1964) The arrangement of chromosomes in the mature sperm of the grasshopper. F. Cell Biol. 21, 286-289.

Tates, A.D., Pearson, P.L. \& Geraedts, J.P.M. (1975) Identification of $X$ and Y spermatozoa in the northern vole, Microtus oeconomus. F. Reprod. Fert. 42, 195-198.

WALKer, M.H. \& MAcGRegor, H.C. (1975) Chromosomes in elongate sperm heads. In Chromosomes Today, Vol. V. Eds P. L. Pearson \& K. R. Lewis. J. Wiley, Jerusalem. 